\title{
Varied Presentations of Non-Hodgkin Lymphoma in Head and Neck Region
}

\author{
Chiranjib Das, ${ }^{1}$ Pritam Chatterjee ${ }^{2}$
}

$\underline{\text { Introduction }}$

\section{$\underline{\text { ABSTRACT }}$}

Majority of lymphomas involving the head and neck are Non-Hodgkin lymphoma (NHL). Most of them present as cervical lymphadenopathy. The objectives of this study are to present the problems encountered in the diagnosis of extra-nodal NHL in head and neck region and show the importance of thorough clinical examination and proper investigation.

Materials And Methods

A prospective study was done in the department of ENT in a tertiary care hospital of West Bengal from July 2015 to June 2018. Patients diagnosed as NHL on the basis of histopathology and immunohistochemistry were included in this study. Patients who were lost in follow up were excluded from the study. Patients were treated with chemotherapy and radiotherapy by Oncologist. All patients were followed up routinely both by Otorhinolaryngologist and Oncologist.

$\underline{\text { Result }}$

There were 26 male and 11 female patients in this study. Patients were between 22 years to 76 years of age with highest incidence in 6th decade of life. Patients presented with only cervical lymphadenopathy; asymmetrical tonsillar enlargement with cervical lymphadenopathy; unilateral tonsillar enlargement only; huge inta-oral mass with stridor; parotid gland swelling; thyroid swelling with multiple cervical lymphadenopathy; epistaxis, palatal ulcer and cheek swelling; severe trismus with inconspicuous retro-mandibular mass. Majority of patients did well with chemoradiotherapy. Two patients succumbed to death, one with huge intra-oral NHL and another with nasal T/NK-cell lymphoma.

Conclusion

Good knowledge of the clinical characteristics of extra-nodal NHL and the methods to establish the diagnosis are essential for a correct and timely therapy of the disease.

Kevwords

Lymphoma, Non-Hodgkin; Head and Neck.

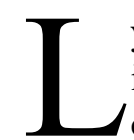
ymphoma is the second most common neoplasm in head and neck region after squamous cell carcinoma. ${ }^{1}$ The majority of lymphomas involving the head and neck are Non-Hodgkin's lymphomas (NHL). ${ }^{2}$ The incidence of NHL has increased up to $35 \%$ in the last 20 years with variation in between different countries. ${ }^{3}$ Most of the NHLs in head and neck region present as cervical lymphadenopathy. But many of them start inside throat especially in the Waldeyer's ring. ${ }^{4} \mathrm{NHL}$ in nose, salivary glands, thyroid and larynx are very rare. ${ }^{5}$

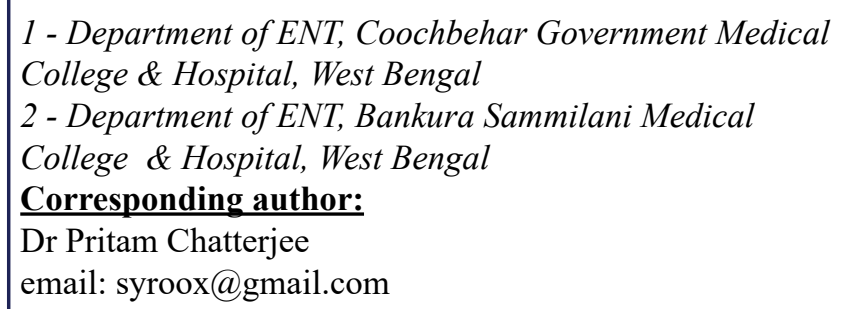

The objectives of this study are to present the problems encountered in the diagnosis of extra-nodal NHL in head and neck region and show the importance of thorough clinical examination and proper investigation.

\section{Materials and Methods}

A prospective study was done in the department of ENT in a tertiary care hospital of West Bengal from July 2015 to June 2018. Patients diagnosed as NHL on the basis of histopathology and immunohistochemistry were included in this study. Patients who were lost in follow up were excluded from the study. After taking proper history, a thorough clinical examination of ear, nose, throat and lympho-reticular system was done. Diagnostic nasal endoscopy and fibre-optic laryngoscopy were done in all patients. Routine haematological tests, 


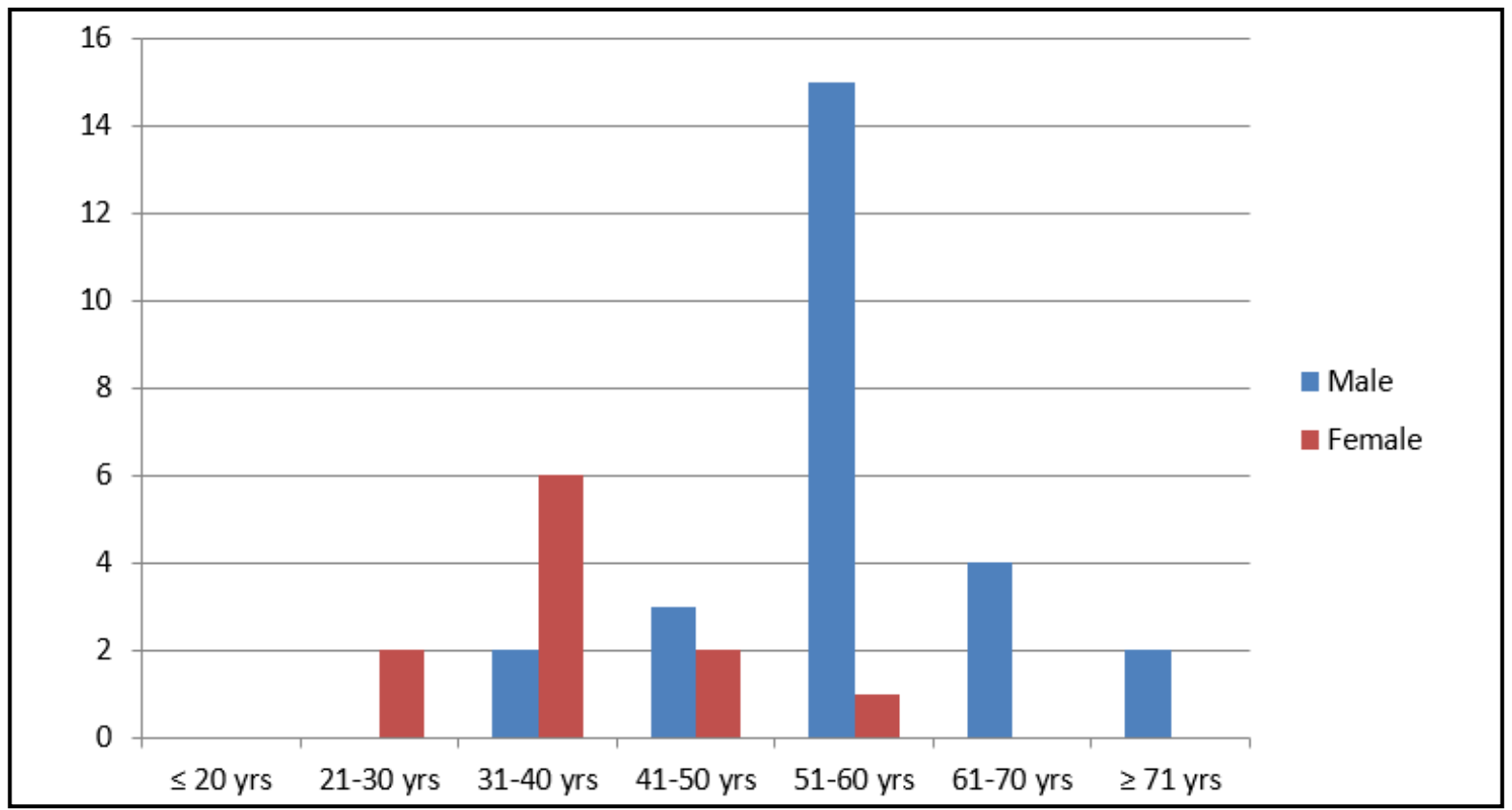

Table I: Distribution of patients according to age and sex

digital chest X-ray, USG whole abdomen were done in all patients. CECT of nose and paranasal sinuses, oral cavity, neck, thorax and abdomen were done whichever was indicated in a particular case. Biopsy was taken in adequate amount meticulously from the involved site and was sent for histopathological examination and immunohistochemistry. Thorough clinical information was given to the pathologist. Repetitive biopsy was needed in some patients to establish a diagnosis. Once the diagnosis of NHL was established, patients were treated with chemotherapy and radiotherapy by Oncologist. All patients were followed up routinely both by ENT surgeon and Oncologist.

\section{Results}

Total 37 patients were diagnosed as NHL in head and neck region over 3 years in the present study. Among them 26 were male and 11 were female. Patients were between 22 years to 76 years of age with highest incidence in 6th decade of life (Table I). 21 out of 37 patients presented with only cervical lymphadenopathy (Table II). 12 patients presented with asymmetrical tonsillar enlargement with cervical lymphadenopathy (Fig. 1). Four patients presented with unilateral tonsillar enlargement only (Fig. 2). Most of them were diagnosed with punch biopsy from tonsil. Only two patients needed tonsillectomy to prove the diagnosis. One patient presented with huge mass inside oral cavity, dysphagia, respiratory distress and stridor (Fig. 3).

Emergency tracheostomy was done followed by wedge biopsy from intra-oral mass. One patient presented with swelling of right parotid gland (Fig. 4). One patient presented with solitary nodule in right lobe of thyroid with multiple cervical lymphadenopathy, huge swelling over sternum, multiple soft tissue nodules in both kidneys and multiple parietal swelling (Fig. 5). One patient presented with recurrent epistaxis, palatal ulcer and swelling over right cheek (Fig. 6).

Histopathogical report of the tissue from nasal cavity was inconclusive. Immunohistochemistry established the diagnosis as T/NK-cell lymphoma. One patient presented with severe trismus without any obvious cause (Fig. 7). On CECT oral cavity, a small heterogenous mass was noticed in right retro-mandibular region. Incision biopsy from the mass established the diagnosis of NHL. Majority of patients did well with chemoradiotherapy. Two patients succumbed to death, one with huge intraoral NHL and another with nasal T/NK-cell lymphoma. 


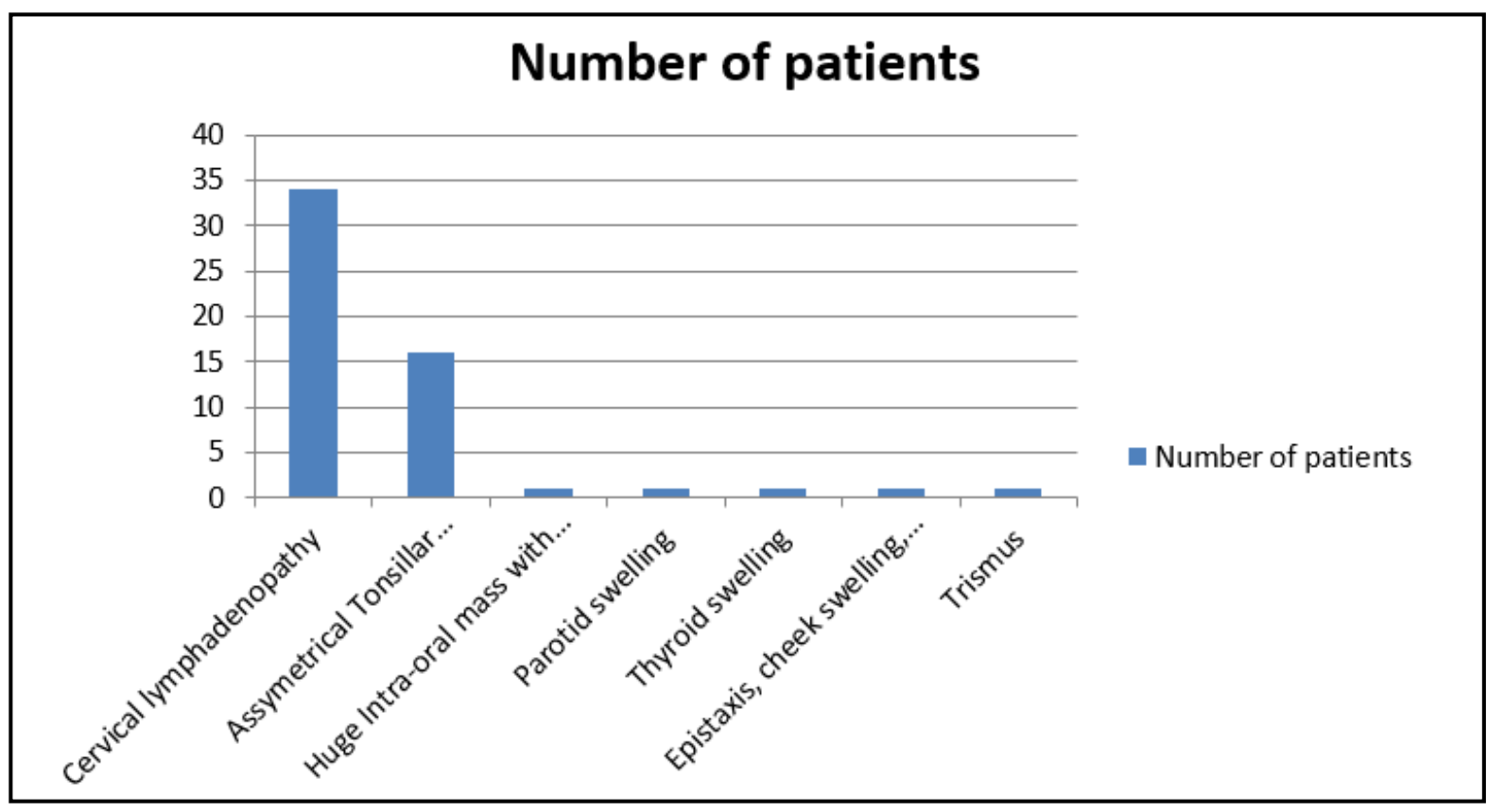

Table II: Distribution of patients according to presentation

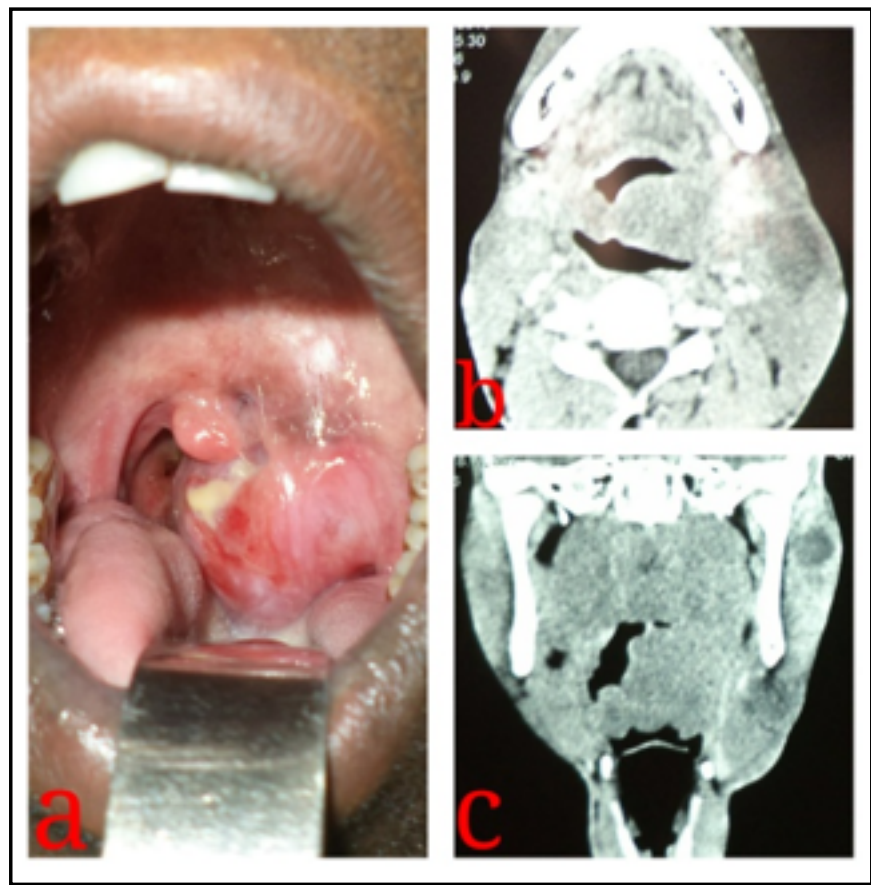

Fig. 1. (a) Clinical photograph showing left tonsillar swelling. (b) CECT neck axial section and (c) CECT neck coronal section showing enlarged cervical lymph node along with left tonsillar swelling.

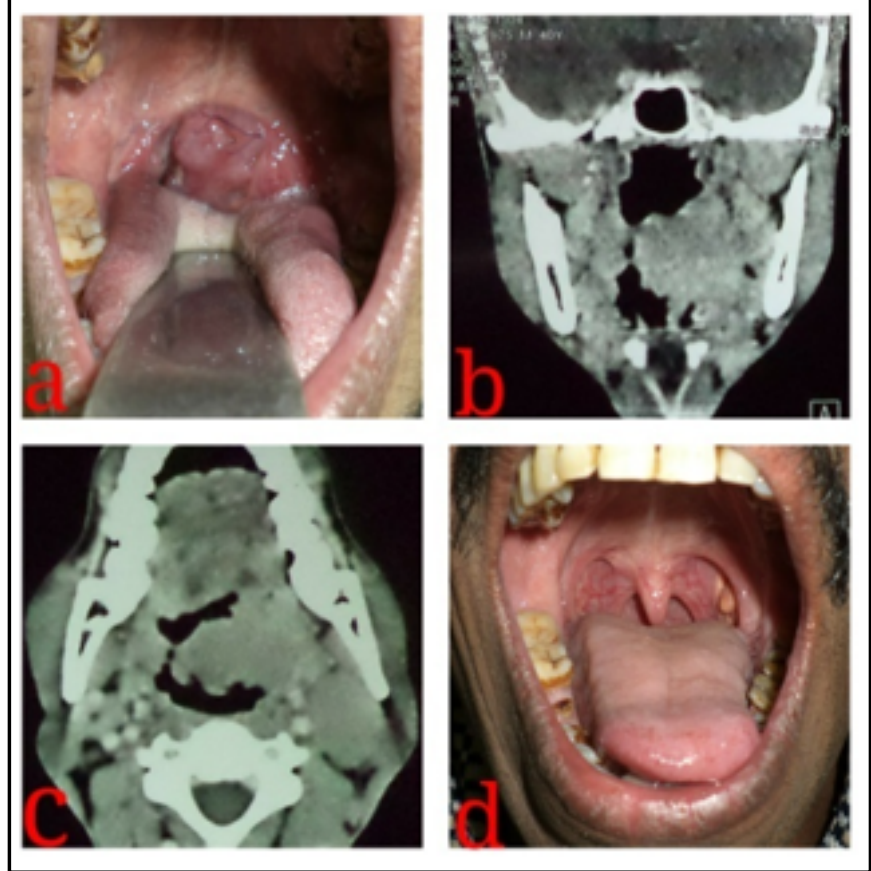

Fig. 2. (a) Clinical photograph showing left tonsillar swelling. (b) CECT neck coronal section and (c) CECT neck axial section showing left tonsillar swelling. (d) Post-treatment clinical photograph showing complete 

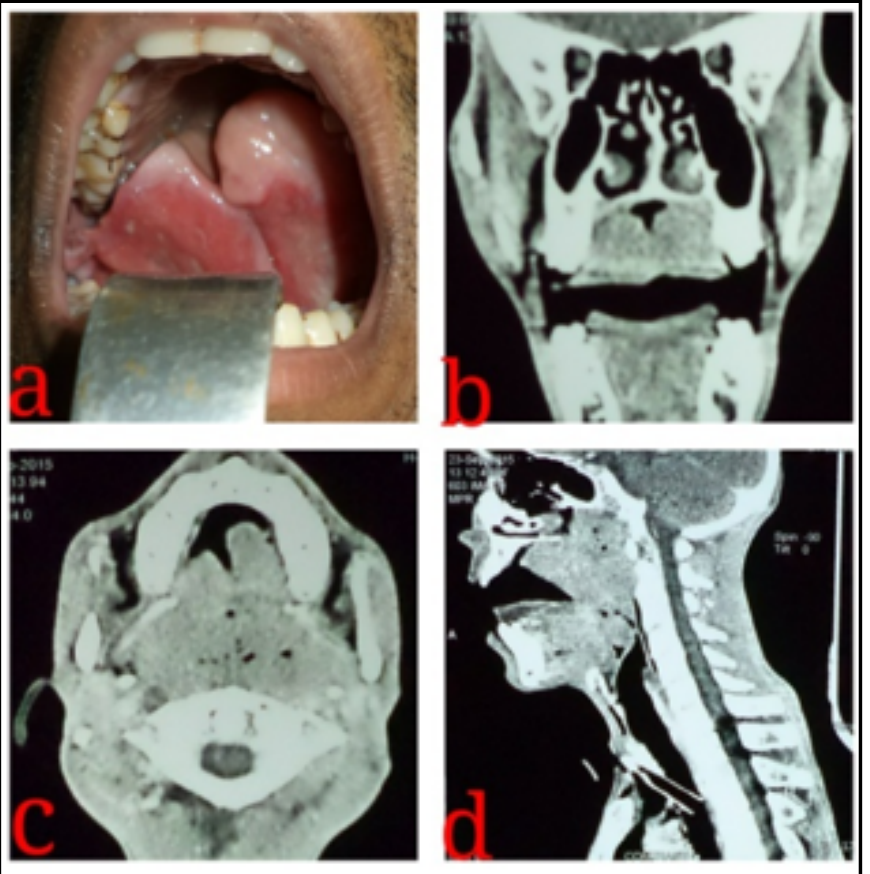

Fig. 3. (a) Clinical photograph showing huge intra-oral mass. (b) CECT nose, PNS and oral cavity coronal section, (c) CECT neck axial section and (d) CECT neck sagittal section showing huge mass occupying nasopharynx, oropharynx and oral cavity extwnding to parapharyngeal spaces with tracheostomy tube in situ.

\section{Discussion}

Lymphomas are malignant neoplasms of lymphocytes and their precursors. ${ }^{6}$ Until 1990, different classifications were used. In 1994 a new classification was implemented called REAL (Revised European American Lymphoma Classification). Based on this, the current WHO classification was developed and is generally used. ${ }^{7}$ Approximately $10 \%$ of lymphomas are Hodgkin lymphoma (HL) and $90 \%$ are Non-Hodgkin lymphoma (NHL). ${ }^{3}$ NHLs usually develop in lymph nodes. But about one third of the NHLs are extra-nodal. ${ }^{8}$ Head and neck is the second most common region for the extra-nodal lymphomas after gastrointestinal tract. ${ }^{9}$ About $10 \%$ of extra- nodal lymphomas are seen in the head and neck region. ${ }^{10}$ The localization of this tumour reveals tonsil to be the most common, followed by nasopharynx, oral cavity, salivary glands, paranasal sinuses and base of tongue. ${ }^{9}$

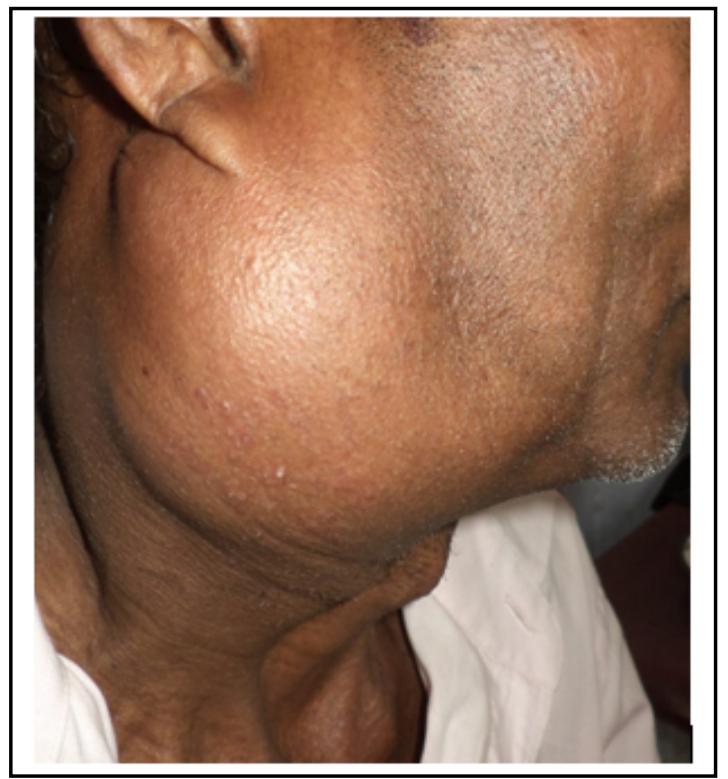

Fig. 4: Clinical photograph showing swelling of right parotid gland.



Fig. 5. (a) Clinical photograph showing thyroid swelling, cervical lymphadenopathy and swelling over sternum. (b) CECT neck axial section showing enlarged right lobe of thyroid with ill-defined non-enhancing hypodense SOL (c) CECT thorax axial section showing swelling over sternum. 


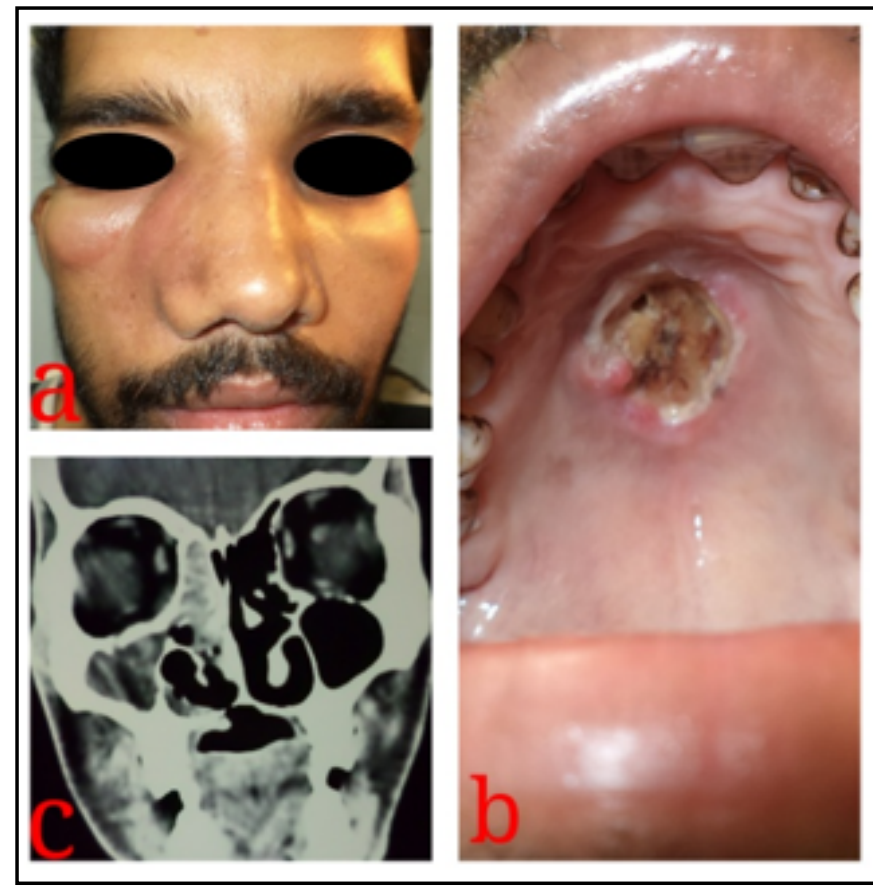

Fig. 6. (a) Clinical photograph showing swelling over right cheek (b) clinical photograph showing palatal ulcer in midline eroding underlying bone. (c) CECT nose and PNS showing destruction of hard palate and right inferior turbinate with mild soft tissue opacity in right nasal cavity and right maxillary sinus.

The distribution of the age in this study is in accordance with the literature. 3 with most patients being older than 50 years (22 out of 37 patients). Usually NHLs of the head and neck region occur more often in men, with approximately $55-77 \%$ of cases which is similar in the present study $(70.27 \%){ }^{11}$ Diagnosis includes a combination of physical examination, blood tests, diagnostic imaging and selective biopsies. Unfortunately the symptoms of NHLs are not specific. Swelling of the area involved and absence of pain are the two signs and symptoms most often described. Systemic signs and symptoms (fever of unknown origin, inexplicable weight loss and night sweating) are usually more often described in patients with Hodgkin's lymphoma than with Non-Hodgkin lymphoma. ${ }^{12}$

Majority of the head and neck extra-nodal NHLs occur in the Waldeyers ring (ie, the tonsils, nasopharynx and the base of the tongue). ${ }^{9} 40 \%$ to $50 \%$ of them arise in the palatine tonsil. Tonsillar lymphomas occur mostly

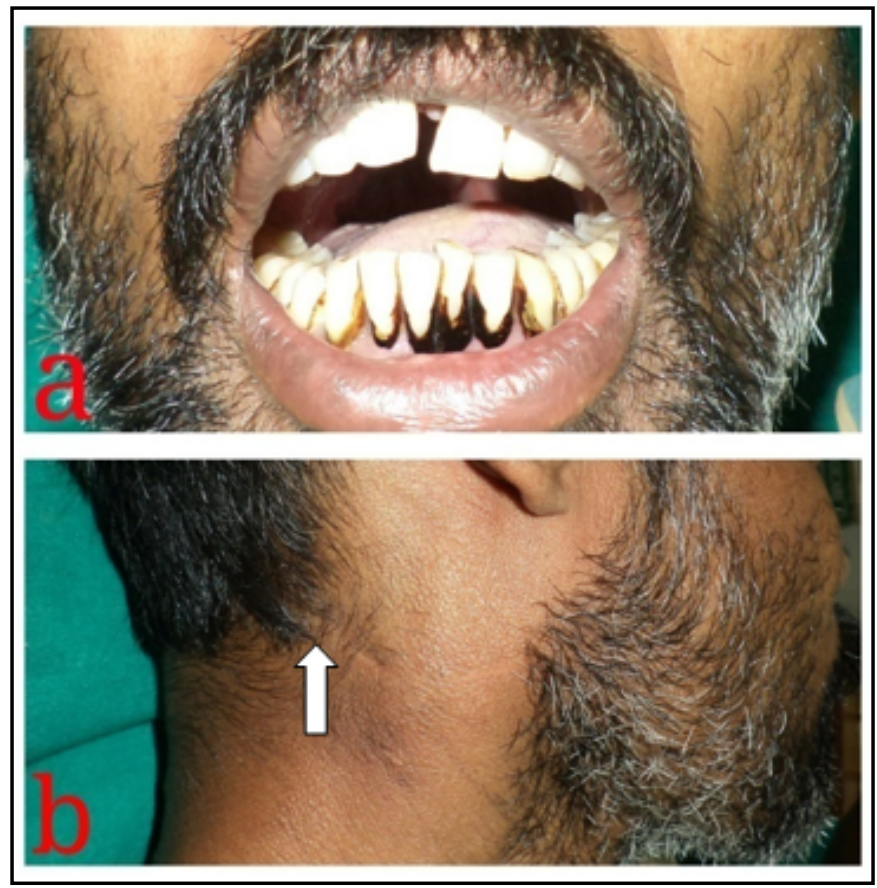

Fig. 7. (a) Clinical photograph showing severe trismus. (b) Clinical photograph showing scar mark of biopsy from inconspicuous retro-mandibular swelling.

in elderly males and present with sore throat, unilateral tonsillar enlargement, cervical lymphadenopathy or dysphagia. ${ }^{6}$ It can easily be mistaken for tonsillitis. Thus, by the time a biopsy is taken, the lymphoma may already be in an advanced, incurable stage..$^{13} 67 \%$ to $96 \%$ of primary tonsillar NHLs are of diffuse large B-cell type (DLBCL). ${ }^{9}$ All patients with tonsillar NHL in this study were of DLBCL type.

The vast majority of lymphomas of salivary glands are located in the parotid..$^{14}$ The patient in this series had low grade B-cell lymphoma of MALT type in right parotid gland. However, the true incidence of primary lymphomas of the salivary glands is difficult to estimate because of the presence of intra-parotid lymph nodes from which nodal lymphomas may arise, despite classification as extranodal. ${ }^{14}$

Lymphoma of the thyroid may either originate in situ or affect the thyroid secondarily as a manifestation of systemic disease. Primary lymphoma of the thyroid 
gland represents $2-5 \%$ of extra-nodal NHLs. Secondary involvement seen in $20 \%$ dying of generalized lymphoma. Regional lymph node enlargement can be seen in some cases. ${ }^{15,16}$ The patient in this study had NHL involving multiple organs that are thyroid, lymph nodes, kidneys.

Primary lymphoma of the nose and paranasal sinuses is very difficult to recognize both clinically and pathologically. These lymphomas appear to be exceedingly rare in Western countries, where they usually show a B-cell phenotype, but relatively common among Asians and Native Americans of Central and South America. Indeed, in Asian countries they represent the second largest group of extra-nodal lymphomas after GI localizations, and most of them have T/NKcell phenotype. Affected patients generally present with nasal obstruction, rhinorrhea or epistaxis. Later on it causes disfigurement, as the nasal bones are eroded. Patients present with destructive nasal or midline facial lesion, erythema, oedema and swelling. This category of lymphoma has been referred to in the past as lethal midline granuloma and, more recently, as angiocentric T/NK-cell nasal lymphoma. Differential diagnosis includes nonspecific sinusitis, rhinitis and Wegener's granulomatosis. ${ }^{17}$ In the present study histopathological examination could not make a definitive diagnosis. Only immunohistochemistry could establish it to be nasal $\mathrm{T} /$ NK-cell lymphoma.

It may be clinically impossible to distinguish the origin of the tumour based on the localization of first symptoms. CT and MRI have greatly facilitated the definition of disease extent, even though documentation of precise margins remains difficult for some localizations (e.g., paranasal sinus, oral cavity). ${ }^{18}$ Computed thomography (CT) of the head and neck, chest, abdomen and pelvis is the mainstay of staging for lymphoma. Bone marrow biopsy is equally mandatory for staging. Concurrent positron emission tomography (PET) with 18F-fluorodeoxyglucose (FDG) and computed tomography (PET/CT) is a useful method for staging and assessment of therapeutic response. ${ }^{19}$

Ambiguous results of histopathological examination should alert the physician of a potential presence of lymphoma. A study by Yen et al. emphasized the importance of repetitive biopsies containing sufficient amounts of tissue for histopathological examination. The tissue should be preserved in buffered formalin and sent to the pathologist immediately after the biopsy. ${ }^{13}$ In case of lymph node biopsy, excision of a intact lymph node should be tried. ${ }^{12}$ Repeated biopsy, even tonsillectomy biopsy is sometimes needed for correct diagnosis of the disease. ${ }^{9}$ Two patients in the present study needed tonsillectomy to come to the diagnosis. Histopathologically, nasal T/NK-cell lymphoma characteristically shows a broad spectrum of cell size, associated with features of angiocentric and angiodestructive growth and extensive necrosis. In early stages, there can be a prominent inflammatory background, often causing difficulty in differentiating it from an inflammatory or infectious process. A simple punch biopsy from the nasal cavity may not yield a satisfactory amount of tissue in majority of the patients. ${ }^{17}$

Careful immunohistochemical assessment is vital in order to differentiate lymphomas from other malignancies as well as to recognize the type of lymphoma. The presence of positive staining for leukocyte common antigen (LCA) in histopathological specimen distinguishes malignant lymphomas from non-lymphoid neoplasms. ${ }^{11}$ Most of lymphomas of the head and neck are aggressive B-cell lymphomas like DLBCL, followed by indolent lymphomas (MALT type and follicular lymphomas). ${ }^{20}$

Mutilating surgery should be avoided. The role of surgery is limited to biopsy. Definitive therapy has to be tailored to the stage and histological subtype. ${ }^{21}$ Prognosis of nasal T/NK-cell lymphoma is usually poor.

\section{Conclusion}

Lymphomas represent approximately $5 \%$ of all malignant neoplasms of the head and neck area. The most common manifestation of NHL in head and neck region is cervical lymphadenopathy, thus making the otolaryngologists the first physicians to deal with it. Most frequent extra-nodal site is the Waldeyer's ring. Good knowledge of the clinical characteristics of these lymphomas and the methods to establish the diagnosis are essential for a correct and timely therapy of the disease. 


\section{References}

1. Shindoh M, Takami T, Arisue M, Yamashita T, Saito T, Kohgo T, Notani K, Totsuka Y, Amemiya A. Comparison between submucosal (extra-nodal) and nodal non-Hodgkin's lymphoma (NHL) in the oral and maxillofacial region. J Oral Pathol Med. 1997; 26:283-9

2. Sasai K, Yamabe H, Kokubo M, et al. Head-and-neck stages I and II extranodal non-Hodgkin's lymphomas: Real classification and selection for treatment modality. Int J Radiat Oncol Biol Phys. 2000; 48:153-60

3. Shankland KR, Armitage JO, Hancock BW. Non-Hodgkin lymphoma. Lancet 2012; 380: 848-57

4. Wang CC. Primary malignant lymphoma of the oral cavity and paranasal sinuses. Radiology 1971; 100:151-3

5. Bloomfield CD, Gajl-Peczalska KJ, Frizzera G, Kersey JH, Goldman AI. Clinical utility of lymphocyte surface markers combined with the Lukes-Collins histologic classification in adult lymphoma. N Engl J Med. 1979; 301:512-8

6. Kolokotronis A, Konstantinou N, Christakis I, Papadimitriou P et al. Localized B-cell non-Hodgkin's lymphoma of oral cavity and maxillofacial region. Oral Surg Oral Med Oral Pathol Oral Radiol Endod. 2005; 99:303-10

7. Swerdlow SH, Campo E, Harris NL, Jaffe ES, Pileri SA, Stein $\mathrm{H}$, et al. WHO Classification of Tumours of Haematopoietic and Lymphoid Tissues. Lyon: IARC; 2008

8. Budhy TI, Soenarto SD, Yaacob HB, Ngeow WC. Changing incidence of oral and maxillofacial tumours in East Java, Indonesia, 1987-1992. Part 2: Malignant tumours. Br J Oral Maxillofac Surg. 2001; 39:460-4

9. Epstein JB, Epstein JD, Le ND, Gorsky M: Characteristics of oral and paraoral malignant lymphoma: a population-based review of 361 cases. Oral Surg Oral Med Oral Pathol Oral Radiol Endod. 2001, 92:519-25

10. Weisenburger DD. Epidemiology of non-Hodgkin's lymphoma: recent findings regarding an emerging epidemic. Ann Oncol. 1994; 5(Suppl 1):19-24
11. Etemad-Moghadam S, Tirgary F, Keshavarz S, Alaeddini M. Head and neck non-Hodgkin's lymphoma: a 20-year demographic study of 381 cases. Int J Oral Maxillofac Surg. 2010; 39:869-72

12. Zapater E, Bagan JV, Carbonell F, Basterra J. Malignant lymphoma of the head and neck. Oral Diseases 2010;16: 119-28

13. Yen TT, Wang RC, Jiang RS, et al. The diagnosis of sinonasal lymphoma: a challenge for rhinologists. Eur Arch Otorhinolaryngol. 2012; 269: 1463-9

14. Harris NL. Lymphoid proliferations of the salivary glands. Am J Clin Pathol. 1999; 111 (Suppl 1): S94-103

15. Hyjek E. Isaacson PG. Primary B-cell lymphoma of the thyroid and its relationship to Hashimoto's thyroiditis. Hum Pathol. $1988 ; 19: 1315-26$

16. Anscombe AN. Wright DH. Primary malignant lymphoma of the thyroid - a tumour of mucosa-associated lymphoid tissue: Review of seventy-six cases. Histopathology 1985; 9: 81-97

17. Jaffe ES, Chan JKC, Su IJ et al. Report of the workshop on nasal and related extranodal angiocentric T/Natural Killer cell lymphomas. Definitions, differential diagnosis, and epidemiology. Am J Surg Pathol. 1996; 20: 103-11

18. Chisin R.Weber AL. Imaging of lymphoma manifestations in the extracranial head and neck region. Leuk Lymph. 1994; 12: 177-89

19. Elstrom RL, Leonard JP, Coleman M, Brown RK: Combined PET and low-dose, noncontrast CT scanning obviates the need for additional diagnostic contrast-enhanced CT scans in patients undergoing staging or restaging for lymphoma. Ann Oncol. 2008, 19(10):1770-3

20. Cortelazzo S, Rossi A, Federico M, et al. The stage modified IBI (MIPI), histology, and combined treatment influence the clinical outcome of 401 patients with primary extranodal head and neck lymphomas (PHNBCL). Blood 2005; 106: Abstract 927

21. Munro A. Lymphomas of the oral cavity and oropharynx. In Stafford N, Waldron J (eds): Management of Oral Cancer. New York: Oxford University Press 1989, 196-200. 